\title{
DRD2/ANKKI Polymorphism Modulates the Effect of Ventral Striatal Activation on Working Memory Performance
}

\author{
Charlotte Nymberg, ${ }^{*, 1}$, Tobias Banaschewski ${ }^{2,3}$, Arun LW Bokde ${ }^{4}$, Christian Büchel ${ }^{5}$, Patricia Conrod ${ }^{6,7}$, \\ Herta Flor ${ }^{2,3}$, Vincent Frouin ${ }^{8}$, Hugh Garavan ${ }^{9,10}$, P Gowland '1, Andreas Heinz ${ }^{12}$, Bernd Ittermann ${ }^{13}$, \\ Karl Mann ${ }^{14}$, Jean-Luc Martinot ${ }^{15,22}$, Frauke Nees ${ }^{2,3}$, Tomas Paus ${ }^{16,17,18}$, Zdenka Pausova' ${ }^{19}$, \\ Marcella Rietschel ${ }^{2,3}$, Trevor W Robbins ${ }^{20}$, Michael N Smolka ${ }^{21}$, Andreas Ströhle ${ }^{12}$, Gunter Schumann ${ }^{6}$, \\ Torkel Klingberg' and the IMAGEN consortium ${ }^{22}$
}

'Department of Neuroscience, Karolinska institute, Stockholm, Sweden; ${ }^{2}$ Department of Child and Adolescent Psychiatry, Central Institute of Mental Health, Mannheim, Germany; ${ }^{3}$ Medical Faculty Mannheim, University of Heidelberg, Mannheim, Germany; ${ }^{4}$ nstitute of Neuroscience and Discipline of Psychiatry, School of Medicine, Trinity College Dublin, Dublin, Ireland; ${ }^{5}$ Department of Systems Neuroscience,

Universitaetsklinikum Hamburg Eppendorf, Hamburg, Germany; ${ }^{6}$ Social Genetic and Developmental Psychiatry Centre, Institute of Psychiatry, King's College London, London, UK; ' Department of Psychiatry, Universite de Montreal, CHU Ste Justine Hospital, Montreal, QC, Canada; ${ }^{8}$ Neurospin, Commissariat à l'Energie Atomique et aux Energies Alternatives, Paris, France; ${ }^{9}$ Institute of Neuroscience, Trinity College Dublin, Dublin, Ireland; ' ${ }^{10}$ Departments of Psychiatry and Psychology, University of Vermont, Burlington, Vermont, USA; " School of Physics and Astronomy, University of Nottingham, Nottingham, UK; ${ }^{12}$ Department of Psychiatry and Psychotherapy, Campus Charité Mitte, Charité- Universitätsmedizin Berlin, Berlin, Germany; ${ }^{13}$ Physikalisch-Technische Bundesanstalt (PTB), Braunschweig und Berlin, Berlin, Germany; ${ }^{14}$ Department of Addictive Behaviour and Addiction Medicine, Central Institute of Mental Health, Medical Faculty Mannheim/Heidelberg University, Mannheim, Germany; ${ }^{15}$ Institut National de la Santé et de la Recherche Médicale, INSERM CEA Unit 1000 'Imaging \& Psychiatry', University Paris Sud, Orsay, France; ${ }^{16}$ Rotman Research Institute, University of Toronto, Toronto, Canada; ${ }^{17}$ School of Psychology, University of Nottingham, Nottingham, UK; ${ }^{18}$ Montreal Neurological Institute, McGill University, Montreal, QC, Canada; ${ }^{19}$ The Hospital for Sick Children, University of Toronto, Toronto, Canada; ${ }^{20}$ Department of Experimental Psychology, Behavioural and Clinical Neurosciences Institute, University of Cambridge, Cambridge, UK; ${ }^{21}$ Department of Psychiatry and Psychotherapy, Neuroimaging Center, Technische Universität Dresden, Dresden, Germany; ${ }^{22}$ AP-HP Department of Adolescent Psychopathology and Medicine, Maison de Solenn, University Paris Descartes, Paris, France

Motivation is important for learning and cognition. Although dopaminergic (D2) transmission in the ventral striatum (VS) is associated with motivation, learning, and cognition are more strongly associated with function of the dorsal striatum, including activation in the caudate nucleus. A recent study found an interaction between intrinsic motivation and the DRD2/ANKKI polymorphism (rs I800497), suggesting that A-carriers of rs 1800497 are significantly more sensitive to motivation in order to improve during working memory (WM) training. Using data from the two large-scale imaging genetic data sets, IMAGEN ( $n=1080$, age $13-15$ years) and BrainChild ( $n \sim 300$, age 6-27), we investigated whether rs 800497 is associated with WM. In the IMAGEN data set, we tested whether VS/caudate activation during reward anticipation was associated with WM performance and whether rs 1800497 and VS/caudate activation interact to affect WM performance. We found that rs 1800497 was associated with WM performance in IMAGEN and BrainChild. Higher VS and caudate activation during reward processing were significantly associated with higher WM performance $(p<0.000 \mathrm{I})$. An interaction was found between the DRD2/ANKKI polymorphism rs 800497 and VS activation during reward anticipation on WM ( $p<0.0$ I), such that carriers of the minor allele (A) showed a significant correlation between VS activation and WM, whereas the GG-homozygotes did not, suggesting that the effect of VS BOLD on WM is modified by inter-individual genetic differences related to D2 dopaminergic transmission.

Neuropsychopharmacology (20I4) 39, 2357-2365; doi:I0.I038/npp.20I4.83; published online I4 May 20I4

*Correspondence: Dr C Nymberg, Department of Neuroscience, Karolinska institute, Retzius v $\tilde{A}$ gg 8, Stockholm 17177, Sweden, Tel: +46727033334, Fax: +468333864, E-mail: charlotte.nymberg@ki.se

${ }^{22}$ See appendix.

Received 20 December 2013; revised 14 March 2014; accepted 14 March 2014; accepted article preview online 9 April 2014

\section{INTRODUCTION}

Dopamine is known to affect working memory (WM) performance as well as processes relating to reward and motivation, which may indirectly influence WM performance (Vijayraghavan et al, 2007; Williams and GoldmanRakic, 1995). Density of dopamine transporters and receptors in the brain have been shown to affect WM performance (Backman et al, 2011) and recent research 
shows that dopaminergic genes known to affect receptor densities in the brain also influence WM (Söderqvist et al, 2013). Here, we investigate whether the DRD2/ANKK1 polymorphism and it's interaction with striatal activation reflecting levels of motivation affect WM performance.

Density of D2 receptors in the striatum have been shown to affect motivation, as shown in a study of rodents where postsynaptic D2 receptor overexpression in the ventral striatum (VS) increased the animal's motivation in terms of willingness to expend effort to obtain a goal (Trifilieff et al, 2013). Dopaminergic neurotransmission is also central for WM performance (Aalto et al, 2005). In humans, cortical dopamine release is observed during the performance of WM tasks (Aalto et al, 2005). The value of cortical D1 density (McNab et al, 2009) and D2 binding in the striatum for WM has been established using positron emission tomography (Backman et al, 2011).

A widely studied dopaminergic polymorphism affecting reward processes and cognition is the DRD2/ANKK1 variant rs1800497 (Glu ${ }^{713} \mathrm{Lys}$ ). The polymorphism is known to be in linkage disequilibrium with the DRD2 gene (Neville et al, 2004) and is believed to affect dopamine binding in the striatum amongst healthy volunteers (Hirvonen et al, 2004). A recent study from our group showed that the DRD2/ ANKK1 polymorphism is associated with WM performance after cognitive training. Furthermore, an interaction between DRD2/ANKK1-genotype and measured intrinsic motivation (ie, engaging in a task for the sake of pure interest) on WM improvement was identified after WM training. The results suggest an association between intrinsic motivation and improvement during WM training amongst minor allele-carriers (A) of rs1800497 (Söderqvist et al, 2013) (Figure 1). Previous research suggest that individuals who carry the A-allele show a reduced density of D2 receptors in the striatum and are more likely to develop disorders of reward deficiency such as alcohol dependence, smoking, and show ADHD symptoms (Blum et al, 1996; Jonsson et al, 1999; Tripp and Wickens, 2008). This might

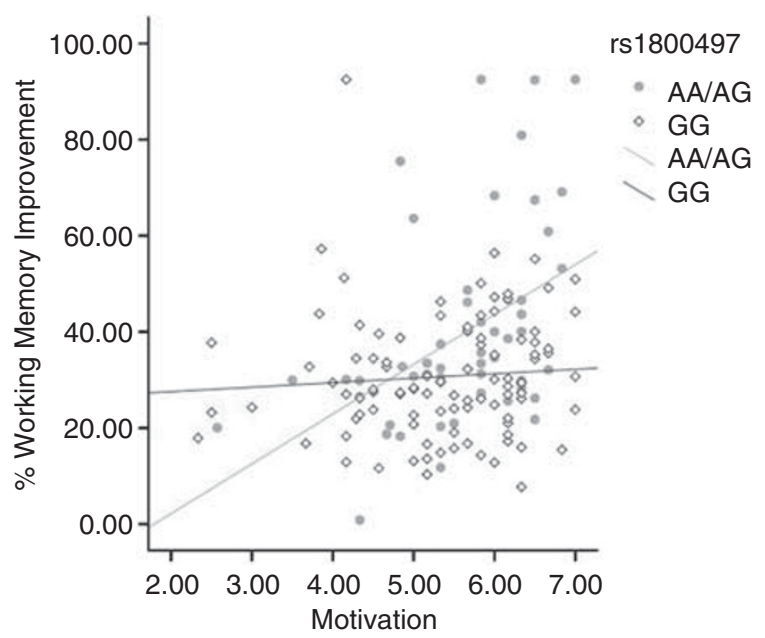

Figure I The estimated perceived competence measured by the intrinsic motivation inventory is shown on the $x$-axis in relation to actual improvements in working memory of training. Data points and line fittings are illustrated according to rs 1800497 genotypes. This figure is adapted for purposes of this paper based on data from a sample presented in Söderqvist et al (2013). indicate a higher sensitivity to motivational factors amongst A-carriers through striatal dopamine levels. Following up on the study by Söderqvist et al (2013), we aimed to investigate whether brain activity in the VS and caudate reflecting motivational drive as measured by the monetary incentive delay (MID) task is associated with WM performance.

The striatum, particularly the VS, is the neural substrate mediating feelings of reward and motivation. Single-cell recordings in macaque monkeys show that neurons in the VS are activated in response to external rewards (eg, fruit juice) (Schultz et al, 1997), consistent with previous research of rodents (Wise and Bozarth, 1984). Following up on these results, functional MRI studies of humans suggest that the VS responds to extrinsic rewards such as money or candy (Diekhof et al, 2012; Knutson et al, 2001b; Luking and Barch, 2013). These studies show that the expectation of a reward given successful performance on a task, elicit VS, and particularly nucleus accumbens, activity proportional to the amount of reward at stake (Knutson et al, 2001a; Schott et al, 2008). The role of the VS during WM tasks has been investigated in recent studies suggesting that the VS is more strongly activated to correct than incorrect responses during a WM task (Satterthwaite et al, 2012) and that it may have an important role in tracking responses (Chatham and Badre, 2013). The VS also has an important role during tasks that require intrinsic motivation (Murayama et al, 2010; Wolf et al, 2011).

Caudate BOLD is primarily involved in instrumental learning as well as response inhibition and WM (Durston et al, 2002; Mcnab and Klingberg, 2008) including development of WM during childhood (Darki and Klingberg, 2014; Ullman et al, 2014). In reward-related contexts, the caudate is suggested to have a role in maintaining the reward outcomes of actions to optimize future choices potentially leading to rewards (O'Doherty et al, 2004). The caudate also has a role in learning and memory, where a more active role in obtaining the reward is taken by the participant (Postle and D'Esposito, 1999; Ziermans et al, 2012).

Several imaging studies have investigated brain activity during WM (Beck et al, 2010; Taylor et al, 2004) while simultaneously modulating levels of reward. In general, rewards during WM tasks increases activity in dorsolateral prefrontal and parietal cortex. However, these findings have not explored whether inter-individual differences in brain activity during a motivational task is associated with WM performance. So although the increase in brain activity induced by motivation results in faster reaction times (Beck et al, 2010), changes in response bias (Taylor et al, 2004), and better recall in long-term memory tasks (Pochon et al, 2002), it has not been shown whether the increase in striatal or cortical activity is related to improved performance in terms of higher accuracy in WM trials or generally improved performance on cognitive tasks. Furthermore, genetic variations in COMT and MAOA have been associated with VS BOLD (Camara et al, 2010; Nymberg et al, 2013a), but these studies did not make any connection with WM and it is unclear whether the effect of rewardrelated brain activity on WM is moderated by genetic variations in dopaminergic genes.

Using two independent data sets, the international IMAGEN study and the Swedish BrainChild cohort, we investigate genetic and neural mechanisms that may affect 
WM. The IMAGEN study is composed of genetic, neuroimaging, and behavioral data from 2000 13-15-year-old adolescents, whereas the BrainChild data set is composed of genetic, neuroimaging, and cognitive data from $\sim 300$ children, adolescents, and young adults, age 6-27 years. Whereas the caudate is activated during performance of WM tasks, VS BOLD, reflecting motivational drive has not been associated with WM performance. As previous studies suggest that D2 receptor densities in the caudate and VS differentially affect motivation, with D2 receptor density in the VS being of greater importance for motivation, we aim to establish whether the DRD2/ANKK1 genes, known to affect D2 receptor density, also have differential effect on VS and caudate BOLD (Trifilieff et al, 2013). Our hypothesis was that the interaction between DRD2/ANKK1 polymorphism and motivation on WM training (Söderqvist et al, 2013) would be seen in an equivalent interaction with rewardrelated activity in VS, but not caudate. We furthermore hypothesized that the behavioral effect previously observed during training would be observed in WM performance.

\section{MATERIALS AND METHODS}

\section{Imagen}

\section{Participants}

Behavioral and neuroimaging data. The IMAGEN sample is comprised of 2000 adolescents that were tested at eight assessment centres (London, Nottingham, Dublin, Berlin, Hamburg, Paris, and Dresden)(Schumann et al, 2010). In this study, 1080 participants ( $48 \%$ males, $14.4 \pm 0.4$ years, 13.3-15.4 years (mean age $\pm S D$, range)) were included based on the following criteria: (1) had complete, quality controlled MRI data from the reward anticipation phase of the MID task ( $n=1472)$; (2) had complete, quality controlled cognitive data and intelligence quotient (IQ) data based on the Wechsler intelligence scale for children (WISC) $(n=1338)$; and (3) had complete genetic data for DRD2/ANKK1 Taq1a SNP rs1800497 ( $n=1080$; GG: $n=669$; AA/AG: $n=441 ; 4.9 \%$ of sample were AA-homozygous). Information regarding exclusion criteria and movement outliers is available in Supplementary Information. This study is based on results from Söderqvist et al (2013) who divided genotype groups into AA/AG versus GG-homozygotes; thus, we merged the AA and AG groups. Previous analyses of the IMAGEN data set have addressed questions regarding reinforcement-related behavior. These studies particularly target questions of how brain function affects normal and pathological behavior in adolescence. For reviews on the IMAGEN data set, please see (Nymberg et al, 2013b; Schumann et al, 2010).

Genetic data. Deoxyribonucleic acid was extracted from blood samples. The DRD2/ANKK1 Taq1a genotype is located on chromosome 11q23.1, rs1800497. Genotyping procedures have been previously published (Nymberg et al, 2013a). Information regarding genotyping is available in the Supplementary Information. Means and SD of cognitive behavior, divided by genotype are available in Table 1 .

\section{Cognitive tests}

Visuospatial WM. WM was assessed through the CANTAB (Beck et al, 2010). Within this computerized task,
Table I Demographics

\begin{tabular}{lccl}
\hline & AA/AG $(\boldsymbol{n}=\mathbf{4 4 I})$ & $\mathbf{G G}(\boldsymbol{n}=\mathbf{6 6 9})$ & $\boldsymbol{p}$-value \\
\hline Working memory errors & $19.32(\mid 3.6)$ & $17.27(\mid 2.7)$ & 0.012 \\
ADHD symptoms & $2.84(2.26)$ & $2.67(2.21)$ & 0.25 \\
Conduct symptoms & $1.57(1.50)$ & $1.57(1.57)$ & 0.78 \\
Emotional problems & $1.93(2.00)$ & $1.87(2.08)$ & 0.59 \\
Verbal IQ & $|1| 3.1(\mid 4.6)$ & $112.6(14.5)$ & 0.38 \\
Reasoning IQ & $108.8(13.3)$ & $109.6(13.5)$ & 0.25 \\
\hline
\end{tabular}

Abbreviations: ADHD, attention deficit hyperactivity disorder; $\mathrm{IQ}$, intelligence quotient; WISC, Wechsler intelligence scale for children; WM, working memory. Means and SD of A-carriers and GG-homozygotes on the WM task of the CANTAB, the Strength and Difficulties Questionnaire, and WISC. Differences between the genotype groups are shown under $p$-value.

subjects were required to search through boxes that appear on the screen with the aim to find a blue token hidden inside. After two practice trials with two boxes there were four test trials with each of two, three, four, six, and eight boxes. If the participant returns to an empty box that has already been opened this constitutes a 'between search error'. Thus, in this task we measure the number of errors that participants make. A lower number indicates better performance.

Intelligence. IQ was assessed using the WISC (Wechsler, 1974).

\section{Magnetic resonance imaging}

Functional MRI task. The participants performed a modified version of the MID task to study neural responses to reward anticipation (Knutson et al, 2001b). This eventrelated task consisted of 66 10-s trials. In each particular trial, participants were presented with one of three cue shapes, with 22 trials of each type (cue, $250 \mathrm{~ms}$ ), denoting whether a target (a white square) would subsequently appear on the left or right side of the screen and whether 0 , 2 , or 10 points could be won in that particular trial. After a variable delay (4000-4500 ms) of fixation on a white crosshair, participants were instructed to respond by pressing a button with their left or right index finger as soon as the target appeared. Feedback on whether and how many points were won during the trial was presented for $1450 \mathrm{~ms}$ after the response. Using a tracking algorithm, task difficulty (ie, target duration varied between 100 and $300 \mathrm{~ms}$ ) was individually adjusted such that each participant successfully responded on $\sim 66 \%$ of trials. Participants had first completed a practice session outside the scanner (for $\sim 5 \mathrm{~min}$ ), during which they were instructed that for each five points won they would receive one food snack in the form of small chocolate candies. The current study used the contrast 'anticipation high win versus no win'. Only successful 'hit' trials were included for analysis.

MRI data acquisition. Structural and functional MRI data were acquired at eight IMAGEN assessment sites with 3T MRI scanners of different manufacturers (Siemens, Philips, General Electric, Bruker). The scanning variables were specifically chosen to be compatible with all scanners. 
The same scanning protocol was used at all sites. In brief, high-resolution T1-weighted 3D structural images were acquired for anatomical localization and co-registration with the functional time series $(\mathrm{TR}=2300 \mathrm{~ms}, \mathrm{TE}=2.8 \mathrm{~ms}$, $256 \times 256 \times 160$ matrix, voxel size: $1.1 \times 1.1 \times 1.1)$. T2*weighted images were acquired with a gradient-echo, echoplanar imaging (EPI) sequence. For the MID task, 300 volumes $\times 40$ slices were acquired for each subject $(2.4 \mathrm{~mm}$ slice thickness, $1 \mathrm{~mm}$ gap). The echo-time was optimized to provide reliable imaging of subcortical areas $(\mathrm{TR}=2200 \mathrm{~ms}$, $\mathrm{TE}=30 \mathrm{~ms}, 64 \times 64$ matrix, voxel size: $3 \times 3 \times 3 \mathrm{~mm}$ ).

MRI analysis. Preprocessing and statistical analysis of fMRI data were performed in SPM8 (Statistical Parametric Mapping, http://www.fil.ion.ucl.ac.uk/spm). Slice-time correction was conducted to adjust for time differences owing to multislice-imaging acquisition; all volumes were aligned to the first volume and non-linear warping was performed to an EPI template. Images were then smoothed with a Gaussian kernel of 5-mm full-width at half-maximum. At the first level of analysis, changes in the BOLD-response for each subject were assessed by linear combinations at the individual subject level; for each experimental condition, each trial was convolved with the hemodynamic response function to form regressors that account for potential noise variance associated with the processing of reward anticipation. Estimated movement parameters were added to the design matrix in the form of 18 additional columns (three translations, three rotations, three quadratic and three cubic translations, and each three translations with a shift of \pm 1 TR). Furthermore, each individual fMRI time series underwent an automatic spike detection method. The spike detector algorithm uses a mean-squared-based metric to identify unexpected values temporally and spatially slice per slice. We used a non-parametric outlier detection procedure based on density estimation to detect and exclude individuals with contrast maps that exceeded criterion values in cortical and subcortical regions (Fritsch et al, 2012). These outlier criteria are used in previous studies, for eg, see (Loth et al, 2013; Nees et al, 2012). Controlling for cortical/subcortical outlier variables in the model did not affect the results and there are no genotype differences in the cortical or subcortical outlier variables $(p<0.10)$. To analyze the anticipation phase, we contrasted 'anticipation of high win (here signaled by a circle) versus anticipation of no win (here signaled by a triangle)'. The individual contrast images were subsequently included in a second-level random effects analysis. The VS (MNI: $\pm 14,8$, $-8,9 \mathrm{~mm}$ radius) was extracted using the MarsBar toolbox (http://marsbar.sourceforge.net) as defined by (O'Doherty et al, 2004; Schneider et al, 2012). The left and right caudate was extracted based on the automated anatomical labellings (Tzourio-Mazoyer et al, 2002). Averaged beta-values based on all voxels in the ROIs were used for all analyses (Figure 2).

Analysis. Analyses were performed in SPSS version 21. All results are based on the full model, ie, CANTAB WM = Striatal BOLD + rs1800497 + Striatal BOLD $\times$ rs1800497+ covariates (centre and gender). In all analyses the VS and caudate are analyzed separately. Significance levels related to MRI results are corrected for these two regions. Owing to the high correlation between left and right VS and left and right caudate BOLD responses, we have not corrected for multiple testing based on hemisphere.

\section{Brainchild}

Participants

Behavioral data. Behavioral data were collected at two time points. The first round comprised 331 individuals a

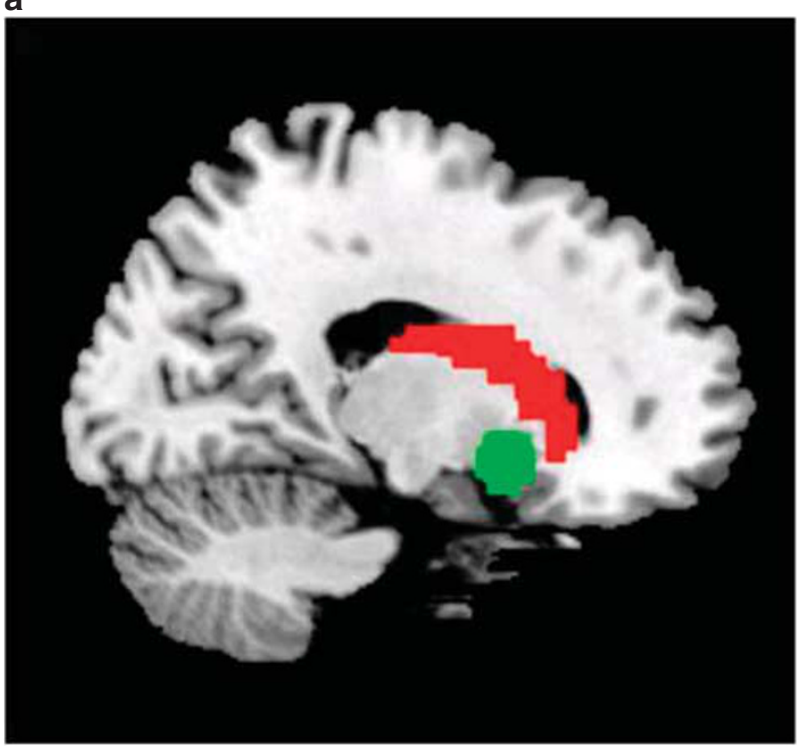

b
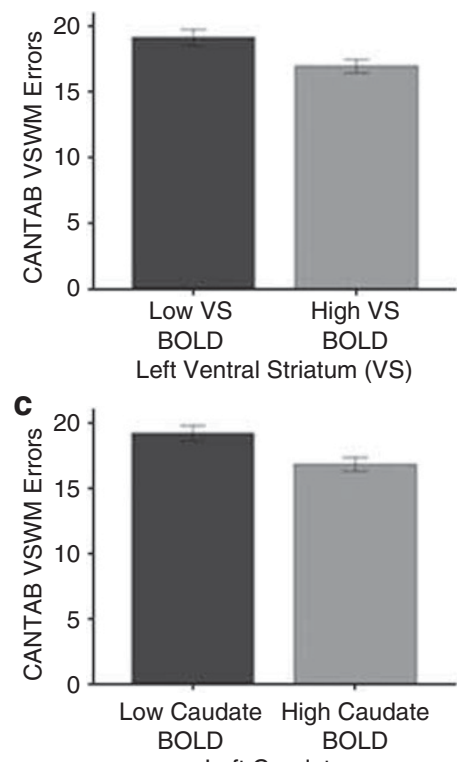

Left Caudate

Figure 2 (a) Sagittal slice indicating caudate region of interest (ROI) in red and VS ROI in green $(x=-\mid 6)$; (b, c) individuals with lower VS/caudate blood oxygenation level dependent (BOLD) defined through a median split of BOLD responses during reward anticipation make significantly more errors on a WM test relative to individuals with high VS/caudate BOLD. 
(50.5\% male, 12.2 years $\pm 4.5,6-25$ years (mean age $\pm S D$, range) ) and the second round 270 individuals (48.9\% male, $13.7 \pm 4.0$ years, $7-27$ years (mean age $\pm S D$, range)). The second round took place 2 years after the first testing session. In round 2, measurements of 256 participants tested at the first instance were repeated. Participants from rounds 1 and 2 were included in analyses using a mixed model ANOVA, as described in the analysis section below. The participants had been recruited using random sampling from the population registry in Nynäshamn in Sweden. The study was approved by the local ethics committee of the Karolinska University Hospital, Stockholm.

Genetic data. Deoxyribonucleic acid was extracted from either blood or saliva samples. Genotyping procedures have been previously published (Dumontheil et al, 2011).

\section{Cognitive tests}

Visuospatial WM. WM performance was assessed using the Automated Working Memory Assessment (AWMA) battery (Dumontheil and Klingberg, 2012). The task involves remembering the location and order of dots displayed sequentially in a four-by-four grid for $1000 \mathrm{~ms}$ each, with a $500 \mathrm{~ms}$ interval between dots. After training with one, two, and three dots the test started with one dot. Each level consisted of six trials. Four correct answers were required for moving to the next level, where one more dot needed to be remembered. The test ended when three errors were committed on one level. The score used was the total number of correct trials. Thus, a higher score is better.

Intelligence. IQ was assessed through Raven's progressive matrices (Raven et al, 1998).

Analysis. Analyses were performed in SPSS version 21. A mixed linear model was performed using the repeated covariance type 'compound symmetry'. The time point at which data had been collected (T1 or T2) was entered as a repeated variable. WM performance acted as the dependent variable, whereas rs1800497-genotype, age, and IQ were entered as predictor variables.

\section{RESULTS}

\section{Effect of rs1800497 on WM}

Imagen. The effect of rs1800497 on the CANTAB WM between search errors was analyzed with a general linear model, controlling for sex and centre effects. This analysis suggested that GG-homozygotes produced significantly fewer errors, ie, showed higher $\mathrm{WM}$ performance $\left(\mathrm{F}_{1,1070}=\right.$ 6.39, $p<0.012$, partial $\left.\eta^{2}: 0.006\right)$. The association between rs1800497 and WM slightly increased when also adding verbal IQ (based on the WISC) as a covariate $\left(\mathrm{F}_{1,1067}=7.39\right.$, $p<0.007$, partial $\left.\eta^{2}: 0.007\right)$.

Brainchild. Linear mixed models with age ${ }^{-1}$, sex, and rs1800497-genotype entered as covariates were used to test for genetic effects on performance on the AWMA. An effect of rs1800497-genotype was identified, with a significantly higher performance on the AWMA identified in individuals homozygous for the G-allele $\left(F_{1,315.7}=6.66, p=0.010\right)$. The SNP by age interaction on AWMA performance was not significant $(p=0.74)$. The association between rs1800497 and WM remained significant when adding IQ-scores in the form of Ravens as a covariate $\left(\mathrm{F}_{1,310.4}=6.42, p=0.036\right)$.

\section{Effect of rs1800497 on BOLD-Response}

Imagen. No direct effects of the rs1800497 polymorphism on either VS (left VS: $p=0.50$, right VS: $p=0.18$ ) or caudate activation (left caudate: $p=0.94$, right caudate: $p=0.29$ ) were identified when controlling for centre, gender, and handedness. No significant genotype differences were identified in the number of successfully completed trials $(p>0.14)$, suggesting that the tracking algorithm of the MID task was effective.

\section{Effect of rs1800497, Striatal BOLD, and rs $1800497 \times$ Striatal BOLD on WM}

Imagen. Next, we tested the full model of rs1800497+ striatal BOLD during reward anticipation $+\operatorname{rs} 1800497 \times$ striatal BOLD during reward anticipation on WM. Four models were completed as left VS, right VS, left caudate, and right caudate varied as predictor variables (Figure $3 \mathrm{~b}$ ). Considering that we tested two regions (VS and caudate), we report corrected significance levels.

\section{Main Effect of rs1800497 on WM}

The effect of rs1800497 on WM was significant also in these models $\left(p<0.0001\right.$, partial $\left.\eta^{2}: 0.012-0.015\right)$.

\section{Main Effect of Striatal BOLD-Response on WM}

A positive BOLD-response was identified in both the VS and the caudate during the contrast anticipation high reward anticipation versus no reward. Increased activation of the VS during this contrast was associated with better WM (measured as between search errors) (left VS $\mathrm{V}_{\text {corrected: }}$ $\mathrm{F}_{1,1068}=21.97, p<0.0001$, partial $\eta^{2} 0.020$; right $\mathrm{VS}_{\text {corrected }}$ : $\mathrm{F}_{1,1068}=16.84, p<0.0001$; partial $\left.\eta^{2}: 0.016\right)$ after controlling for centre and gender. The associations remained significant after adding verbal IQ as a covariate (left $\mathrm{VS}_{\text {corrected: }}$ $\mathrm{F}_{1,1067}=20.57, p<0.0001$, partial $\eta^{2}: 0.019$; right $\mathrm{VS}_{\text {corrected }}$ : $\mathrm{F}_{1,1067}=16.17, p<0.0001$, partial $\eta^{2}: 0.015$ ) (Figure 2). Similarly, increased BOLD in the caudate during reward anticipation was associated with better performance on the CANTAB WM task (left caudate corrected $\mathrm{F}_{1,1068}=18.38, p<0.0001$, partial $\eta^{2}: 0.017$; right caudate corrected $\mathrm{F}_{1,1068}=16.05, p=0.00013$, partial $\left.\eta^{2}: 0.015\right)$. Again, these associations remained significant after controlling for verbal IQ (left caudate corrected: $_{\text {: }}$ : $\mathrm{F}_{1,1072}=18.03, \quad p<0.0001, \quad$ partial $\eta^{2}: 0.017$; right caudate corrected $_{1,1067}=15.51, p<0.00017$, partial $\left.\eta^{2}: 0.014\right)$.

\section{Rs1800497 $\times$ Striatal BOLD-Response Interaction on WM}

We identified a significant interaction between rs1800497 and $\mathrm{VS}_{\text {corrected }}$ BOLD on WM (left $\mathrm{VS}_{\text {corrected }} \times$ rs1800497: $\mathrm{F}_{1,1068}=10.48, p=0.002$, partial $\eta^{2}: 0.010$; right $\mathrm{VS}_{\text {corrected }} \times$ rs1800497: $\quad \mathrm{F}_{1,1068}=7.25, \quad p=0.014$, partial $\left.\eta^{2}: 0.007\right)$ 


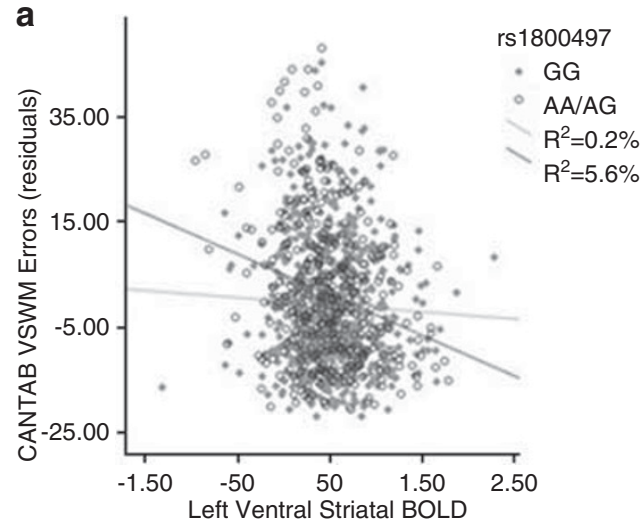

b

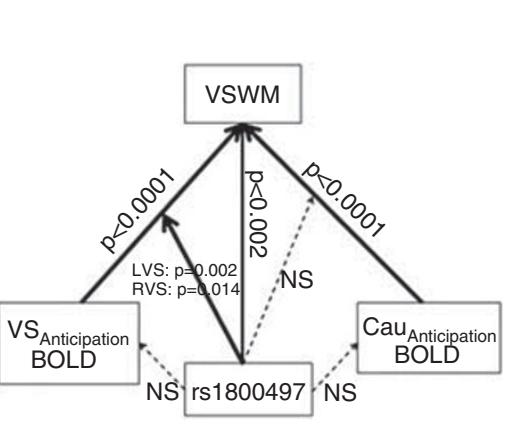

Figure 3 (a) rs $1800497 \times$ ventral striatum (VS) blood oxygenation level dependent (BOLD) interaction suggesting that individuals carrying the A-allele make more errors on a working memory (WM) $\left(r=-0.24, p<0.0001, r^{2}=5.6 \%\right)$ when they have reduced VS BOLD during reward anticipation. The negative correlation was not significant amongst GG-homozygotes $\left(r=-0.04, p=0.27, r^{2}=0.2 \%\right)$. Residuals of WM errors after controlling for centre and gender are shown. (b) Depiction of main effects and interactions from the model rs $\mid 800497+$ striatal $B O L D+r s \mid 800497 \times s t r i a t a l B O L D=W M$ (controlling for centre and gender) based on the IMAGEN sample. The non-significant effects of $r$ I 800497 on BOLD responses are based on the individual analyses. The figure shows significant main effects of rs 1800497 on WM, main effects of caudate and VS BOLD on WM as well as interactions between rs 1800497 and BOLD responses on WM.

suggesting that A-carriers, who on average performed worse on tests of WM, show a significantly higher accuracy on the WM task with higher VS activation (Figure 3). These interactions remained significant in the left VS after covarying for verbal IQ (left $\mathrm{VS}_{\text {corrected }} \times$ rs1800497: $\mathrm{F}_{1,1067}=8.14, p=0.004$, partial $\eta^{2}: 0.008$; right $\mathrm{VS}_{\text {corrected }} \times$ rs1800497: $\quad \mathrm{F}_{1,1067}=4.61, \quad p=0.064$, partial $\left.\eta^{2}: 0.004\right)$. Interactions between caudate BOLD and WM were not significant after correcting for multiple testing (left caudate $_{\text {corrected }} \times$ rs 1800497: $\mathrm{F}_{1,1068}=3.34, \quad p=0.14$; right caudate $_{\text {corrected }} \times$ rs1800497: $\left.\quad \mathrm{F}_{1,1068}=4.92, \quad p=0.054\right)$. Although the main effect of left VS BOLD accounted for $1.4 \%$ of the variance in WM performance in the full sample, when investigating the variance accounted for by left VS BOLD amongst the A-carriers, $5.6 \%$ of the variance is accounted for, whereas only $0.2 \%$ of the variance is accounted for amongst the GG-homozygotes.

\section{DISCUSSION}

The results of this study show that VS and caudate activity during reward anticipation have a small but significant effect on WM performance in a large sample of adolescents. Whereas striatal activity has previously been associated with WM (Satterthwaite et al, 2012; Ziermans et al, 2012), this is the first study to show that striatal activity during a reward task is associated with WM performance measured outside the scanner, suggesting that striatally mediated motivation is important for WM performance (Figure $3 \mathrm{~b}$ ). Furthermore, the study suggests that the DRD2/ANKK1 polymorphism rs1800497, which has previously been shown to interact with motivation to influence improvement after WM training, also interacts with VS activity during reward processing to affect WM performance. Individuals who carry the lower expressed A-allele show reduced WM performance relative to GG-homozygotes. According to previous studies, A-carriers also tend to develop disorders of reward deficiency, such as alcohol abuse, and ADHD more frequently (Blum et al, 1996; Comings and Blum, 2000); here we show that these individuals show a positive association between reward-related VS activity and WM performance.

In a study of a different sample, Söderqvist et al (2013) showed that individuals who carry the A-allele of rs1800497 are more sensitive to intrinsic motivation during WM training in order to improve their WM relative to the GG-homozygotes (Söderqvist et al, 2013). The study suggests that A-carriers improve significantly more on a task of WM after completing several weeks of cognitive training. The sample showed no association between rs1800497 and WM at baseline and the significant interaction between rs1800497 and motivation on WM remained after controlling for baseline performance. Our study suggests that that A-carriers of rs1800497 may also be significantly more sensitive to VS activity during reward processing in order to perform well on a WM task. However, the A-carriers also perform significantly worse on a tasks of WM. Thus, we speculate that the relative performance of A-carriers and GG-homozygotes depends on the amount of activity in VS, and on the level of motivation during testing. Although this association of rs1800497-genotype on WM was not identified in the study by Söderqvist et al (2013), the findings fit well with previous research suggesting that individuals who carry the A-allele show reduced density of D2 receptors in the VS and are more likely to develop disorders of reward deficiency such as excessive drinking, smoking, and show ADHD symptoms (Blum et al, 1996; Jonsson et al, 1999; Volter et al, 2012). We speculate that the reward deficiency, which has previously been associated with A-carriers of rs1800497, does not only affect motivation but indirectly also impacts on WM performance. In our sample, we did not identify significant associations between rs 1800497 and ADHD/drinking behaviors, but we cannot exclude that unknown third variables drive the observed interaction.

In conclusion, these results add clarity to the neural mechanisms that underlie associations between motivation 
and WM. Previous studies implicate striatal function as important for WM. We suggest that striatal BOLD during reward processing is an important factor underlying performance on tasks of WM, but that individuals differ in their response. In combination with the study by Söderqvist et al (2013), this study suggests that individuals who show reduced WM based on ANKK1/DRD2 genotype may be able to increase their performance through increased motivation, which is associated with striatal activation. Future research should aim to investigate the effect of striatal activation during different phases of reward processing on WM performance in an MRI task that integrates both reward processing and WM. It would also be of use to better understand the molecular mechanisms by which rs 1800497 affects D2 receptors in the striatum in humans. Finally, it would be of interest to determine whether motivation during WM training affects striatal activation.

\section{FUNDING AND DISCLOSURE}

The IMAGEN project was supported by the European Union-funded FP6 Integrated Project IMAGEN (reinforcement-related behavior in normal brain function and psychopathology) (LSHM-CT-2007-037286), the FP7 project IMAGEMEND (IMAging GEnetics for MENtal Disorders), and the Innovative Medicine Initiative Project EU-AIMS (115300-2), Medical Research Council Programme Grant 'Developmental pathways into adolescent substance abuse' (93558), as well as the Swedish funding agency FORMAS. Further support was provided by the Bundesministerium für Bildung und Forschung (NGFN Plus; FKZ: 01GS08152) and the Deutsche Forschungsgemeinschaft (DFG): ReinhartKoselleck Award SP 383/5-1. This research was also supported by the German Ministry of Education and Research (BMBF grant no. 01EV0711). CN's postdoctoral appointment is funded by the Wenner-Gren foundation. TB served in an advisory or consultancy role for Hexal Pharma, Lilly, Medice, Novartis, Otsuka, Oxford outcomes, PCM scientific, Shire, and Viforpharma. He received conference attendance support and conference support or received speaker's fee by Lilly, Medice, Novartis, and Shire. He is/has been involved in clinical trials conducted by Lilly, Shire, and Viforpharma. The present work is unrelated to the above grants and relationships. AS received research funding from the German Federal Ministry of Education and Research, the European Commission (FP6), and Lundbeck and speaker honoraria from AstraZeneca, Boehringer, Ingelheim, Bristol-Myers Squibb, Eli Lilly, Pfizer, Lundbeck, Wyeth, and UCB. AS was a consultant for Actelion. Educational grants were given by the Stifterverband für die Deutsche Eissenschaft, the Berlin Brandenburgische Akademie der Wissenschaften, the Boehringer Ingelheim Fonds, the Eli Lilly International Foundation, Janssen-Cilag, Pfizer and Eli Lilly \& Co. AH has received research funding from the German Research Foundation and the Bernstein Center for Computational Neuroscience Berlin (German Federal Ministry of Education and Research), Eli Lilly \& Co, Janssen-Cilag and Bristol-Myers Squibb. AH has received speaker honoraria from Janssen-Cilag, Johnson \& Johnson, Lilly, Pfizer, and Servier. TWR consults for Cambridge Cognition and receives royalties for CANTAB. He also consults for Lilly, Lundbeck, Teva, Shire Pharmaceuticals, and Chempartners and has received research grants from Lilly, Lundbeck, and GlaxoSmithKline. The remaining authors declare no conflict of interest.

\section{REFERENCES}

Aalto S, Bruck A, Laine M, Nagren K, Rinne JO (2005). Frontal and temporal dopamine release during working memory and attention tasks in healthy humans: a positron emission tomography study using the high-affinity dopamine D2 receptor ligand [11C]FLB 457. J Neurosci 25: 2471-2477.

Backman L, Nyberg L, Soveri A, Johansson J, Andersson M, Dahlin E et al (2011). Effects of working-memory training on striatal dopamine release. Science 333: 718 .

Beck SM, Locke HS, Savine AC, Jimura K, Braver TS (2010). Primary and secondary rewards differentially modulate neural activity dynamics during working memory. Plos One 5: e9251.

Blum K, Cull JG, Braverman ER, Comings DE (1996). Reward deficiency syndrome. American Scientist 84: 132-145.

Camara E, Kramer UM, Cunillera T, Marco-Pallares J, Cucurell D, Nager W et al (2010). The effects of COMT (Val108/158Met) and DRD4 (SNP - 521) dopamine genotypes on brain activations related to valence and magnitude of rewards. Cereb Cortex 20: 1985-1996.

Chatham CH, Badre D (2013). Working memory management and predicted utility. Front Behav Neurosci 7: 83.

Comings DE, Blum K (2000). Reward deficiency syndrome: genetic aspects of behavioral disorders. Prog Brain Res 126: 325-341.

Darki F, Klingberg T (2014). The role of fronto-parietal and frontostriatal networks in the development of working memory: a longitudinal study. Cereb Cortex doi:10.1093/cercor/bht352.

Diekhof EK, Kaps L, Falkai P, Gruber O (2012). The role of the human ventral striatum and the medial orbitofrontal cortex in the representation of reward magnitude-an activation likelihood estimation meta-analysis of neuroimaging studies of passive reward expectancy and outcome processing. Neuropsychologia 50: 1252-1266.

Dumontheil I, Klingberg $\mathrm{T}$ (2012). Brain activity during a visuospatial working memory task predicts arithmetical performance 2 years later. Cereb Cortex 22: 1078-1085.

Dumontheil I, Roggeman C, Ziermans T, Peyrard-Janvid M, Matsson H, Kere J et al (2011). Influence of the COMT genotype on working memory and brain activity changes during development. Biol Psychiatry 70: 222-229.

Durston S, Thomas KM, Yang Y, Uluğ AM, Zimmerman RD, Casey BJ (2002). A neural basis for the development of inhibitory control. Dev Sci 5: F9-F16.

Fritsch V, Varoquaux G, Poline JB, Thirion B (2012). Nonparametric density modeling and outlier-detection in medical imaging datasets. In Wang F (Ed.) Machine learning in medical imaging. Springer-Verlag: Berlin Heidelberg, Vol 7588, pp 210-217.

Hirvonen M, Laakso A, Nagren K, Rinne JO, Pohjalainen T, Hietala J (2004). C957T polymorphism of the dopamine D2 receptor (DRD2) gene affects striatal DRD2 availability in vivo. Mol Psychiatry 9: 1060-1061.

Jonsson EG, Nothen MM, Grunhage F, Farde L, Nakashima Y, Propping P et al (1999). Polymorphisms in the dopamine D2 receptor gene and their relationships to striatal dopamine receptor density of healthy volunteers. Mol Psychiatry 4: 290-296.

Knutson B, Adams CM, Fong GW, Hommer D (2001a). Anticipation of increasing monetary reward selectively recruits nucleus accumbens. J Neurosci 21: RC159.

Knutson B, Fong GW, Adams CM, Varner JL, Hommer D (2001b). Dissociation of reward anticipation and outcome with eventrelated fMRI. Neuroreport 12: 3683-3687. 
Loth E, Poline JB, Thyreau B, Jia T, Tao C, Lourdusamy A et al (2013). Oxytocin Receptor genotype modulates ventral striatal activity to social cues and response to stressful life events. Biol Psychiatry doi:10.1016/j.biopsych.2013.07.043.

Luking KR, Barch DM (2013). Candy and the brain: neural response to candy gains and losses. Cogn Affect Behav Neurosci 13: $437-451$.

Mcnab F, Klingberg T (2008). Prefrontal cortex and basal ganglia control access to working memory. Nat Neurosci 11: 103-107.

McNab F, Varrone A, Farde L, Jucaite A, Bystritsky P, Forssberg H et al (2009). Changes in cortical dopamine D1 receptor binding associated with cognitive training. Science 323: 800-802.

Murayama K, Matsumoto M, Izuma K, Matsumoto K (2010). Neural basis of the undermining effect of monetary reward on intrinsic motivation. Proc Natl Acad Sci USA 107: 20911-20916.

Nees F, Tzschoppe J, Patrick CJ, Vollstadt-Klein S, Steiner S, Poustka L et al (2012). Determinants of early alcohol use in healthy adolescents: the differential contribution of neuroimaging and psychological factors. Neuropsychopharmacology 37: 986-995.

Neville MJ, Johnstone EC, Walton RT (2004). Identification and characterization of ANKK1: a novel kinase gene closely linked to DRD2 on chromosome band 11q23.1. Hum Mutat 23: 540-545.

Nymberg C, Jia T, Lubbe S, Ruggeri B, Desrivieres S, Barker G et al (2013a). Neural mechanisms of attention-deficit/hyperactivity disorder symptoms are stratified by MAOA genotype. Biol Psychiatry 74: 607-614.

Nymberg C, Jia T, Ruggeri B, Schumann G (2013b). Analytical strategies for large imaging genetic datasets: experiences from the IMAGEN study. Ann N Y Acad Sci 1282: 92-106.

O’Doherty J, Dayan P, Schultz J, Deichmann R, Friston K, Dolan RJ (2004). Dissociable roles of ventral and dorsal striatum in instrumental conditioning. Science 304: 452-454.

Pochon JB, Levy R, Fossati P, Lehericy S, Poline JB, Pillon B et al (2002). The neural system that bridges reward and cognition in humans: An fMRI study. Proc Natl Acad Sci USA 99: 5669-5674.

Postle BR, D’Esposito M (1999). Dissociation of human caudate nucleus activity in spatial and nonspatial working memory: an event-related fMRI study. Cognitive Brain Res 8: 107-115.

Raven J, Raven JC, Court JH (1998). Manual for Raven's progressive matrices and vocabulary scales. Oxford Psychologists Press: Oxford.

Satterthwaite TD, Loughead J, Ruparell K, Elliott MA, Gerraty RT, Calkins ME et al (2012). Being right is its own reward: load and performance related ventral striatum activation to correct responses during a working memory task in youth. Biol Psychiatry 71: 184s-184s.

Schneider S, Peters J, Bromberg U, Brassen S, Miedl SF, Banaschewski $\mathrm{T}$ et al (2012). Risk taking and the adolescent reward system: a potential common link to substance abuse. Am J Psychiatry 169: 39-46.

Schott BH, Minuzzi L, Krebs RM, Elmenhorst D, Lang M, Winz OH et al (2008). Mesolimbic functional magnetic resonance imaging activations during reward anticipation correlate with reward-

The other members of the IMAGEN consortium (http:// www.imagen-europe.com) are:

King's College, Institute of Psychiatry, London, UK: L Reed, S Williams, A Lourdusamy, S Costafreda, A Cattrell, C Nymberg, L Topper, L Smith, S Havatzias, K Stueber, C Mallik, T-K Clarke, D Stacey, C Peng Wong, H Werts, $S$ Williams, C Andrew, S Desrivieres, S Zewdie (Coordination office). Department of Psychiatry and Psychotherapy, Campus Charité Mitte, Charité Universitätsmedizin Berlin, related ventral striatal dopamine release. J Neurosci 28: 14311-14319.

Schultz W, Dayan P, Montague PR (1997). A neural substrate of prediction and reward. Science 275: 1593-1599.

Schumann G, Loth E, Banaschewski T, Barbot A, Barker G, Buchel $C$ et al (2010). The IMAGEN study: reinforcement-related behaviour in normal brain function and psychopathology. $\mathrm{Mol}$ Psychiatry 15: 1128-1139.

Söderqvist S, Matsson H, Peyrard-Janvid M, Kere J, Klingberg T (2013). Polymorphisms in the dopamine receptor 2 gene region influence improvements during working memory training in children and adolescents. J Cogn Neurosci 26: 54-62.

Taylor SF, Welsh RC, Wager TD, Phan KL, Fitzgerald KD, Gehring WJ (2004). A functional neuroimaging study of motivation and executive function. Neuroimage 21: 1045-1054.

Trifilieff P, Feng B, Urizar E, Winiger V, Ward RD, Taylor KM et al (2013). Increasing dopamine D2 receptor expression in the adult nucleus accumbens enhances motivation. Mol Psychiatry 18: $1025-1033$.

Tripp G, Wickens JR (2008). Research review: dopamine transfer deficit: a neurobiological theory of altered reinforcement mechanisms in ADHD. J Child Psychol Psychiatry 49: 691-704.

Tzourio-Mazoyer N, Landeau B, Papathanassiou D, Crivello F, Etard O, Delcroix N et al (2002). Automated anatomical labeling of activations in SPM using a macroscopic anatomical parcellation of the MNI MRI single-subject brain. Neuroimage 15: 273-289.

Ullman H, Almeida R, Klingberg T (2014). Structural maturation and brain activity predict future working memory capacity during childhood development. J Neurosci 34: 1592-1598.

Vijayraghavan S, Wang M, Birnbaum SG, Williams GV, Arnsten AF (2007). Inverted-U dopamine D1 receptor actions on prefrontal neurons engaged in working memory. Nat Neurosci 10: $376-384$

Volter C, Riedel M, Wostmann N, Aichert DS, Lobo S, Costa A et al (2012). Sensorimotor gating and D2 receptor signalling: evidence from a molecular genetic approach. Int J Neuropsychopharmacol 15: $1427-1440$.

Wechsler D (1974): Wechsler intelligence scale for children (revised. edn). Psychological Corporation, New York.

Williams GV, Goldman-Rakic PS (1995). Modulation of memory fields by dopamine D1 receptors in prefrontal cortex. Nature 376: 572-575.

Wise RA, Bozarth MA (1984). Brain reward circuitry: four circuit elements 'wired' in apparent series. Brain Res Bull 12: 203-208.

Wolf DH, Gerraty RT, Satterthwaite TD, Loughead J, Campellone T, Elliott MA et al (2011). Striatal intrinsic reinforcement signals during recognition memory: relationship to response bias and dysregulation in schizophrenia. Front Behav Neurosci 5: 81.

Ziermans T, Dumontheil I, Roggeman C, Peyrard-Janvid M, Matsson H, Kere J et al (2012). Working memory brain activity and capacity link MAOA polymorphism to aggressive behavior during development. Transl Psychiatry 2: e85.
Berlin, Germany: A Heinz, I Häke, N Ivanov, A Klär, J Reuter, C Palafox, C Hohmann, C Schilling, K Lüdemann, A Romanowski, A Ströhle, E Wolff, M Rapp.PhysikalischTechnische Bundesanstalt, Berlin, Germany: B Ittermann, R Brühl, A Ihlenfeld, B Walaszek, F Schubert. Institute of Neuroscience, Trinity College, Dublin, Ireland: C Connolly, J Jones, E Lalor, E McCabe, A Ní Shiothchái. Department of Psychopharmacology, Central Institute of Mental Health, Mannheim, Germany: R Spanagel, F Leonardi-Essmann, 
W Sommer. Department of Cognitive and Clinical Neuroscience, Central Institute of Mental Health, Mannheim, Germany: S Vollstaedt-Klein. Department of Child and Adolescent Psychiatry, Central Institute of Mental Health, Mannheim, Germany: L Poustka, S Steiner. Department of Addictive Behaviour and Addiction, Medicine, Mannheim, Germany: M Buehler, S Vollstedt-Klein. Department of Genetic Epidemiology in Psychiatry, Central Institute of Mental Health, Mannheim, Germany: E Stolzenburg, C Schmal, F Schirmbeck. Brain and Body Centre, University of Nottingham, Nottingham, UK: P Gowland, N Heym, C Lawrence, C Newman. Technische Universitaet Dresden, Dresden, Germany: T Huebner, S Ripke, E Mennigen, KU Muller, V Ziesch. Department of Systems Neuroscience, University Medical Center Hamburg-Eppendorf, Hamburg, Germany: U Bromberg, T Fadai, L Lueken, J Yacubian, J Finsterbusch. Institut National de la Santé et de la Recherche Médicale, Service Hospitalier Frédéric Joliot, Orsay, France: J-L Martinot, E Artiges, N Bordas, $\mathrm{S}$ de Bournonville, $\mathrm{Z}$ Bricaud, F Gollier Briand, H Lemaitre, J Massicotte, R Miranda, J Penttilä. Neurospin, Commi- ssariat à l'Energie Atomique, Paris, France: A Barbot, Y Schwartz, C Lalanne, V Frouin, B Thyreau. Department of Experimental Psychology, Behavioural and Clinical Neurosciences Institute, University of Cambridge, Cambridge, UK: J Dalley, A Mar, N Subramaniam, D Theobald, N Richmond, $\mathrm{M}$ de Rover, A Molander, E Jordan, E Robinson, L Hipolata, M Moreno, Mercedes Arroyo. University of Sussex, Brighton, UK: D Stephens, $\mathrm{T}$ Ripley, $\mathrm{H}$ Crombag, $\mathrm{Y}$ Pena.Centre National de Genotypage, Evry, France (CNG): D Zelenika, S Heath. German Centre for Ethics in Medicine, Bonn (DZEM), Germany: D Lanzerath, B Heinrichs, T Spranger. Gesellschaft fuer Ablauforganisation m.b.H. (Munich) (GABO), Germany: B Fuchs, C Speiser. Klinik für Kinder- und Jugendpsychiatrie, Zentrum für Psychosoziale Medizin, Universitätsklinikum Heidelberg, Germany: F Resch, J Haffner, P Parzer, R Brunner. Scito, Paris, France: A Klaassen, I Klaassen. PERTIMM, Asnières-Sur-Seine, France: P Constant, X Mignon. NordicNeuroLabs, Bergen, Norway: T Thomsen, S Zysset, A Vestboe. Delosis Ltd, London, UK: J Ireland, J Rogers.

Supplementary Information accompanies the paper on the Neuropsychopharmacology website (http://www.nature.com/npp) 